\title{
Severe retinopathy of prematurity is associated with reduced cerebellar and brainstem volumes at term and neurodevelopmental deficits at 2 years
}

\author{
Femke J Drost ${ }^{1}$, Kristin Keunen ${ }^{1}$, Pim Moeskops ${ }^{2}$, Nathalie HP Claessens ${ }^{1}$, Femke van Kalken ${ }^{1}$, Ivana Išgum ${ }^{2}$, \\ Elsbeth SM Voskuil-Kerkhof ${ }^{3}$, Floris Groenendaal ${ }^{1}$, Linda S de Vries ${ }^{1}$, Manon JNL Benders ${ }^{1}$ and Jacqueline UM Termote ${ }^{1}$
}

BACKGROUND: To evaluate the association between severe retinopathy of prematurity (ROP), measures of brain morphology at term-equivalent age (TEA), and neurodevelopmental outcome.

METHODS: Eighteen infants with severe ROP (median gestational age (GA) 25.3 (range 24.6-25.9 weeks) were included in this retrospective case-control study. Each infant was matched to two extremely preterm control infants $(n=36)$ by GA, birth weight, sex, and brain injury. T2weighted images were obtained on a $3 \mathrm{~T}$ magnetic resonance imaging (MRI) at TEA. Brain volumes were computed using an automatic segmentation method. In addition, cortical folding metrics were extracted. Neurodevelopment was formally assessed at the ages of 15 and 24 months.

RESULTS: Infants with severe ROP had smaller cerebellar volumes ( $21.4 \pm 3.2$ vs. $23.1 \pm 2.6 \mathrm{ml} ; P=0.04)$ and brainstem volumes $(5.4 \pm 0.5 \mathrm{ml}$ vs. $5.8 \pm 0.5 \mathrm{ml} ; P=0.01)$ compared with matched control infants. Furthermore, ROP patients showed a significantly lower development quotient (Griffiths Mental Development Scales) at the age of 15 months (93 \pm 15 vs. $102 \pm 10 ; P=0.01)$ and lower fine motor scores (10 \pm 3 vs. $12 \pm 2 ; P=0.02)$ on Bayley Scales (Third Edition) at the age of 24 months.

CONCLUSION: Severe ROP was associated with smaller volumes of the cerebellum and brainstem and with poorer early neurodevelopmental outcome. Follow-up through childhood is needed to evaluate the long-term consequences of our findings.

$\mathbf{R}$ etinopathy of prematurity (ROP) is a retinal vasoproliferative disease of the very preterm infant and a major cause of blindness in children (1-3). It is thought to account for $\sim 13 \%$ of childhood blindness in developed countries (3).

When infants are born prematurely, the retina is not fully vascularized. Insulin-like growth factor-1 (IGF-1) and vascular endothelial growth factor (VEGF) are two essential growth factors that need to be in balance for normal retinal vessel growth (4). Peri- and postnatal infections, postnatal undernutrition, and fluctuating oxygen levels-as a result of an immature respiratory system and neonatal illness-may impede IGF-1 production in the preterm infant (5). Concomitantly, VEGF is relatively suppressed by higher oxygen levels present in the extrauterine environment. The combination of VEGF suppression and IGF-1 reduction may result in loss of retinal blood vessels and delayed vessel growth, leaving the peripheral retina avascular. This vasoobliterative period is called the first phase of ROP. Later during the second phase of ROP, hypoxia in the avascular retina upregulates VEGF, resulting in neovascularization on the verge of the vascularized and avascularized retina, forming the most important risk factor for permanent visual impairment due to retinal detachment (4). Increasing IGF-1 concentrations, due to catch up growth of the preterm infant in this period, contribute to this uncontrolled neovascularization (5).

Hence, the retina undergoes substantial development during the extrauterine third trimester that is susceptible to disturbances. Similarly, the cerebral cortex and cerebellum develop rapidly during this period and are vulnerable to disruptive environmental factors (6). Furthermore, brain development is dependent on IGF-1 (ref. 7). Animal studies have revealed its importance in angiogenesis, neurogenesis, maturation, proliferation, differentiation, and myelination $(7,8)$. Given their overlap in timing of rapid growth and development as well as the common role of IGF-1 (refs 9-11), there may be a relationship between ROP and morphological brain development, which has not been studied so far.

In this case-control study, we investigated the association between severe ROP and morphological brain development assessed using magnetic resonance imaging (MRI) at a termequivalent age (TEA). To this end, we compared brain volumes of major brain structures and measures of cortical morphology between preterm infants with severe ROP and

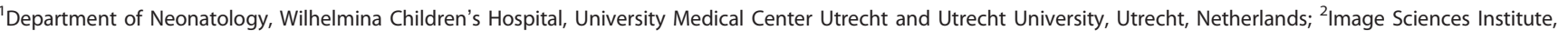
University Medical Center Utrecht and Utrecht University, Utrecht, Netherlands; ${ }^{3}$ Department of Ophthalmology, University Medical Center Utrecht and Utrecht University, Utrecht, Netherlands. Correspondence: Jacqueline U.M. Termote (J.Termote@umcutrecht.nl) 


\section{ROP, brain volumes, and neurodevelopment $\mid$ Articles}

control infants who were matched by gestational age (GA), birth weight (BW), and sex. We hypothesized that preterm infants with ROP would exhibit smaller brain volumes and reduced complexity of cortical gyrification compared with preterm control infants. As a secondary objective, we studied the relationship between severe ROP and neurodevelopmental outcome evaluated at the age of 15 months and 2 years' corrected age (CA). Cognitive and motor performances were hypothesized to be poorer in infants with severe ROP.

\section{METHODS}

\section{Study Population}

In this case-control study, 18 infants with severe ROP were included. Severe ROP was defined as ROP treated with laser therapy, and included grade 3 with plus disease or type 1 ROP. Diagnosis of ROP was first made by an ophthalmologist when all infants born $<30$ weeks' GA are routinely screened for this condition. Infants with ROP were selected from a large population of preterm infants who had been admitted to the level-three NICU of the Wilhelmina Children's Hospital, University Medical Center Utrecht, the Netherlands, between June 2009 and June 2015 and who had a good-quality cerebral MRI at TEA. For each ROP patient, two control infants were selected and matched by GA, BW, sex, year of birth, and brain injury defined as intraventricular hemorrhage grade I and II, diagnosed with cranial ultrasound or MRI according to Papile et al. (12). Matching was performed using propensity scores in SPSS (Armonk, NY, USA). Infants with severe brain lesions defined as intraventricular hemorrhage grade $\geq$ III or cystic periventricular leukomalacia and those with chromosomal anomalies, genetic syndromes, inborn errors of metabolism, and/or congenital infections of the central nervous system were excluded. The total study population consisted of 18 patients with severe ROP and 36 controls without ROP $(n=27)$ or with mild ROP (grade $1, n=9)$. The Institutional Review Board of our institution granted permission for using the clinically acquired data for research purposes. Data were analyzed anonymously and written informed consent was waived by the Institutional Review Board because of the use of clinically acquired, anonymous data.

\section{Procedure}

MRI. MRI examinations were performed on a 3-tesla system at TEA as part of routine clinical care. Prior to scanning, infants were sedated with chloralhydrate $(50-60 \mathrm{mg} / \mathrm{kg}$ ) administered through a nasogastric tube as per the clinical protocol. During the MRI procedure, hearing protection was provided using two pairs of earmuffs (Minimuffs, Natus Medical Incorporated, San Carlos, CA, and Em's 4 Kids, Culver City, CA). Heart rate, transcutaneous oxygen saturation, and respiratory rate were continuously monitored, and a neonatologist or physician assistant was present throughout the entire procedure.

During the 35-min scanning session, coronal T2-weighted images (repetition time $=4,847 \mathrm{~ms}$, echo time $=150 \mathrm{~ms}$, acquired voxel size: $0.89 \times 0.78 \times 1.2 \mathrm{~mm}^{3}$, and reconstructed voxel size: $0.35 \times 0.35 \times 1.2 \mathrm{~mm}^{3}$ ), sagittal T2-weighted images (SENSE single shot, repetition time $=15,000 \mathrm{~ms}$, echo time $=90 \mathrm{~ms}$, voxel size $0.94 \times 1.22 \times 2.00$, slice gap $2 \mathrm{~mm})$, and coronal T1-weighted images (repetition time $=9.5 \mathrm{~ms}$, echo time $=4.6 \mathrm{~ms}$, voxel size $\left.=0.91 \times 0.78 \times 1.2 \mathrm{~mm}^{3}\right)$ were acquired. Cerebellar hemorrhages and white matter injury (evaluated according to Woodward et al. (13) and Kidokoro et al. (14)) were assessed using both T1- and T2-weighted images. Transcerebellar diameter was measured on coronal T2-weighted images as previously described by Kidokoro et al. and Tich et al. $(14,15)$. A representative example of twodimensional transcerebellar measurements is provided in Figure 1.

Brain volumes and cortical folding parameters. Unmyelinated white matter (UWM), cortical grey matter (CoGM), cerebellum, brainstem

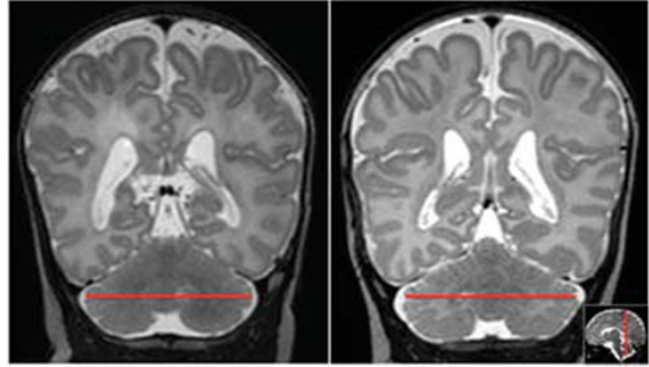

Figure 1. Transcerebellar diameter. Examples of the transcerebellar diameter as measured on a coronal T2-weighted image (left an example of an infant with severe ROP, right a control infant). Measurements were performed at the level of the lateral ventricles where the choroid plexus was clearly visible. ROP, retinopathy of prematurity.

(BS), ventricular and extracerebral cerebrospinal fluid volumes (ml) were automatically segmented on coronal T2-weighted images using an automated segmentation method that employed convolutional neural networks. The segmentation method is described in detail in Moeskops et al. (16). From the UWM and CoGM segmentation, the following cortical folding parameters were calculated: inner CoGM surface area in $\mathrm{mm}^{2}$-defined as the border between the UWM and the CoGM segmentations-and the gyrification index, which is computed as the ratio between the inner CoGM surface and a smooth hull around the UWM segmentation (17). All segmentations were visually inspected for quality assurance, and minor manual adaptations were performed in case of voxel misclassification. Absolute volumes as well as relative volumes-computed as the percentage of intracranial volume of each brain volume-were used for the analysis. Cerebrospinal fluid volumes in the ventricles and surrounding the brain were combined for the analysis (from here on referred to as CSF). Representative examples of brain tissue segmentations are provided in Figure 2.

Neurodevelopmental outcome. Neurodevelopmental outcome was assessed at 15 months' (15 ROP infants and 30 controls) CA using the Griffiths Mental Developmental Scales, which comprises the following five subscales: locomotor function, personal social functioning, hearing and language, eye and hand coordination, and performance. For each infant, a development quotient (mean and SD in a normative population $100 \pm 12$ ) was derived from these subscales. At 2 years' CA, cognitive, fine, and gross motor functioning were assessed using the Bayley Scales of Infant and Toddler Development, Third Edition (BSITD-III; normative mean in a Dutch reference population $100 \pm 15$; ROP $n=15$, controls $n=30)(18)$. For three ROP infants, outcome data at the age of 15 and 24 months were not available.

\section{Statistical Analysis}

Statistical procedures were performed using R (www.r-project.org) and SPSS statistics (IBM, Armonk, NY). Baseline characteristics between ROP patients and controls were compared using independent $t$-tests for normally distributed variables and Mann-Whitney $U$-tests and $\chi^{2}$-tests for non-normally distributed or categorical variables. Multivariable pairwise regression models were undertaken to compare brain volumes, descriptors of cortical folding, transcerebellar diameter, and neurodevelopmental outcome between the two groups, entering ROP as a variable of interest.

Baseline characteristics were compared and variables that were significantly different between the groups were entered as covariates in the model. In case of multicollinearity, the variable that was most likely related to ROP based on literature findings was selected.

Therefore, bronchopulmonary dysplasia (BPD), defined as need of supplemental oxygen at 36 weeks' postmenstrual age (PMA), and multiple birth were included in de model. Neurodevelopmental 

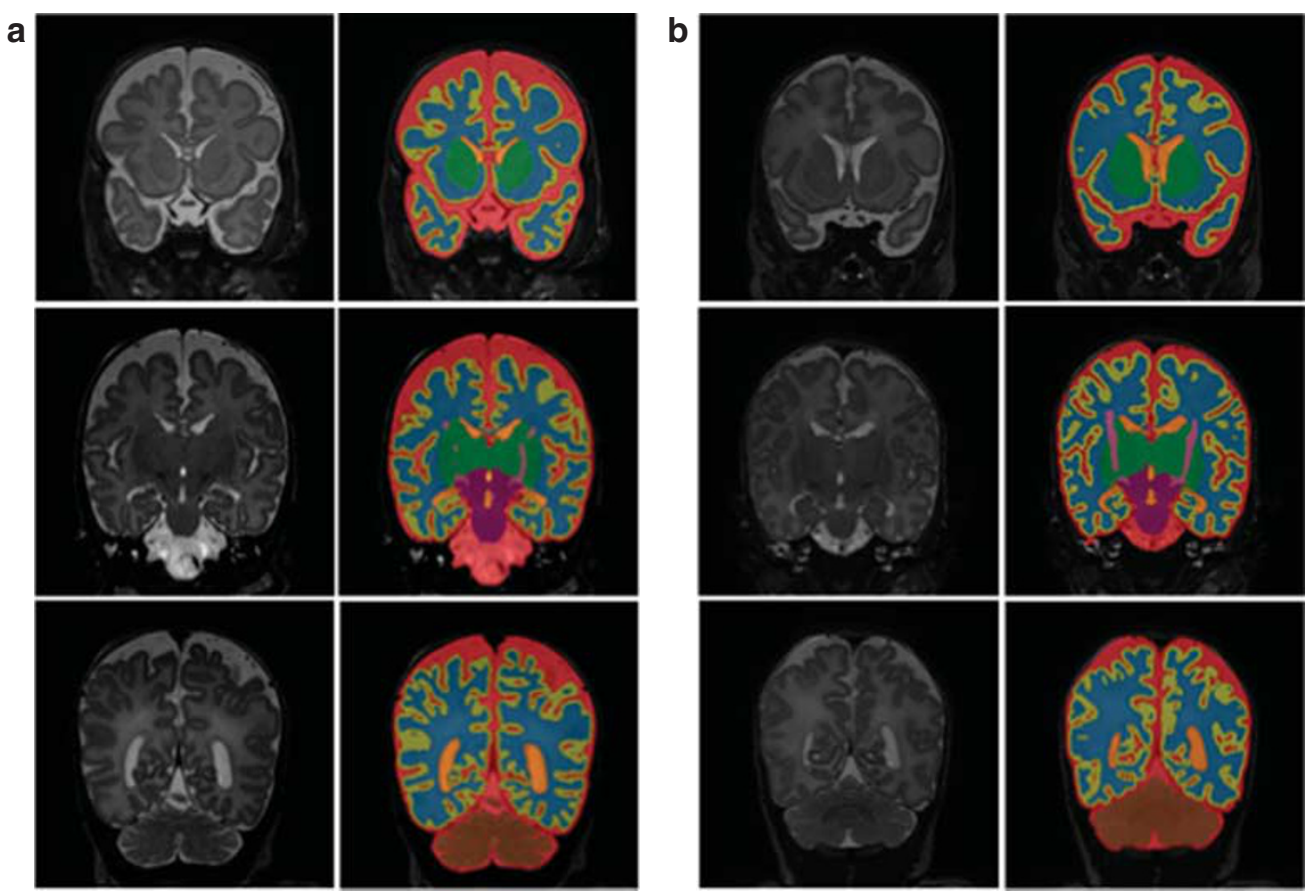

Figure 2. Coronal T2-weighted MRI segmentation. Representative examples of segmentation results of an infant with severe ROP (a) and control infant (b). Left panels show T2-weighted images of a representative infant included in the study. Right panels show corresponding automatic segmentation results of unmyelinated white matter (blue), cortical gray matter (yellow), cerebellum (brown), brainstem (purple), ventricles (orange), and cerebrospinal fluid (red). Basal ganglia and thalami (green) and myelinated white matter (pink) were automatically segmented by this method, but not used for analysis. ROP, retinopathy of prematurity.

outcome data were corrected for age at time of assessment. As maternal educational level has a significant impact on cognitive functioning, especially in infancy and early childhood (19), we compared the level of maternal education between the groups before analyses of neurodevelopmental outcome. No significant differences were found between ROP patients and controls. Therefore, this factor was not entered as a covariate in the multivariate model. Normally distributed variables are listed as their mean and SD and variables that were not normal distributed are provided as median and interquartile range in the Results section. A two-sided $P$ value $<0.05$ was considered statistically significant.

\section{RESULTS}

Clinical characteristics of the study population are outlined in Table 1. There were indeed no significant differences in GA, BW, sex, and intraventricular hemorrhage between infants with severe ROP and control infants as infants were matched. BPD was more common in ROP infants (14 (78\%) vs. 11 (31\%) infants, $\left.P=0.001, \chi^{2}=10.8\right)$. ROP infants received significantly more erythrocyte transfusions (8 (6-10) vs. 5 (3$7), P<0.0001)$ and there were more multiple births in the ROP group (10 (56\%) vs. $\left.7(19 \%), P=0.03, \chi^{2}=7.3\right)$.

\section{Brain Volumes and Cortical Folding}

Preterm infants with severe ROP showed significantly smaller absolute cerebellar volumes $(P=0.04,21.4 \pm 3.2$ vs. $23.1 \pm 2.6 \mathrm{ml})$ and relative cerebellar volumes $(P=0.048$, $4.7 \pm 0.14 \%$ vs. $5.1 \pm 0.09 \%)$ compared with controls. BS volumes were also significantly smaller in ROP infants: absolute BS volume, $P=0.01,5.4 \pm 0.5 \mathrm{ml}$ vs. $5.8 \pm 0.5 \mathrm{ml}$, and relative BS volume, $P=0.02,1.2 \pm 0.03 \%$ vs. $1.3 \pm 0.1 \%$. Results are illustrated in Figure 3. In addition, infants with severe ROP showed a significantly smaller transcerebellar diameter $(P=0.04,5.0 \pm 0.06$ vs. $5.2 \pm 0.06 \mathrm{~cm})$. Entering BPD or multiple births in the model had no significant effect on the association between severe ROP and brain volumes or transcerebellar diameter. No significant differences in CoGM volumes, UWM volume, and CSF volumes were observed between ROP infants and controls. In addition, there were no significant differences in measures of cortical development including cortical surface area and the gyrification index.

\section{Neurodevelopmental Outcome}

ROP patients showed a significantly lower development quotient on the Griffiths Mental Developmental Scales at 15 months' CA $(P=0.01,93 \pm 15$ vs. $102 \pm 10)$. Furthermore, ROP patients exhibited significantly lower fine motor scores $(P=0.02,10 \pm 3$ vs. $12 \pm 2)$ on the BSITD-III at 2 years' CA. No significant differences were observed in cognitive and gross motor scores at the age of 2 years. Outcome data are presented in Table 2. BPD and multiple births did not significantly influence the relationship between severe ROP and neurodevelopmental outcome.

\section{Visual Outcome}

In this cohort visual outcome data were available for $16 / 18$ $(89 \%)$ infants with severe ROP and no data were available for control infants. Fourteen infants had no visual impairment, 


\section{ROP, brain volumes, and neurodevelopment $\quad$ Articles}

Table 1. Clinical characteristics

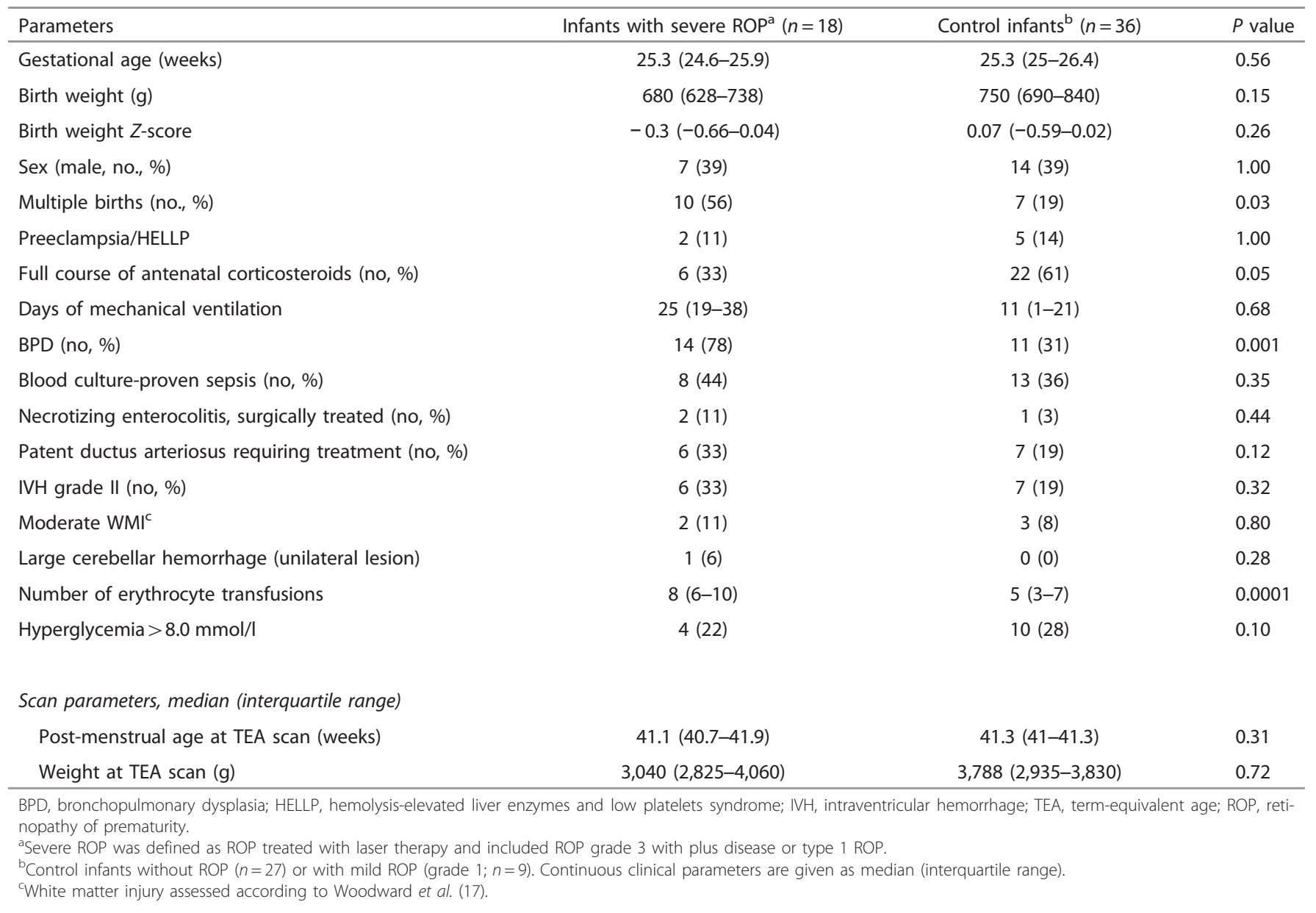

two infants suffered from unilateral blindness, and $44 \%$ of the infants (7/16) were older than 4 years at the age of ophthalmological follow-up. One of the visually impaired ROP infants was not included in the neurodevelopmental outcome analysis because neurodevelopmental outcome could not be assessed. After excluding the visually impaired infant from the analyses, fine motor scores remained significantly lower in infants with severe ROP compared with preterm control infants.

\section{DISCUSSION}

In this case-control study we observed smaller volumes of the cerebellum and associated smaller transcerebellar diameter at TEA in preterm infants with severe ROP compared with control infants who were matched by GA, BW, and sex. Furthermore, ROP patients had significantly smaller BS volumes and poorer early neurodevelopmental outcome.

To our knowledge, this is the first study to make direct comparisons of early brain growth and neurodevelopment between infants with severe ROP and preterm infants without this complication. A Swedish study showed low IGF-1 serum concentrations to be associated with reduced cerebellar volume in preterm infants (9). Although direct comparisons cannot be made, there may be substantial overlap between the observations of the latter study and the present study. IGF-1 is involved in the pathophysiology of ROP as well as in brain growth, and development may have an important mechanistic role in the association between ROP and brain development.

Retinal vessels emerge from the fourth month of pregnancy under control of IGF-1 and VEGF in particular, and are typically mature by 36-40 weeks' gestation (20). Concomitantly, the brain-and specifically the cerebellum-develops rapidly, which makes it vulnerable to environmental changes that may occur during this critical period. Such changes include a rapid fall in IGF-1 after preterm birth and persistent low IGF-1 concentrations in the weeks thereafter.

We hypothesize that disruptions in IGF-1 may be the common underlying pathway for abnormal development of the retina, cerebellum, and BS in the preterm period, when these processes take place in an extrauterine environment. During the first phase of ROP, retinal vessel outgrowth is suppressed because of low levels of IGF-1 (and VEGF). During the second vasoproliferative phase, neovascularizations are formed because of retinal hypoxia causing increased VEGF release. During this phase, IGF-1 levels should have reached sufficient values for vascular growth. IGF-1 is also essential for the developing brain and has been demonstrated to be important for axon maturation and myelination in the 


\section{Articles | Drost et al.}
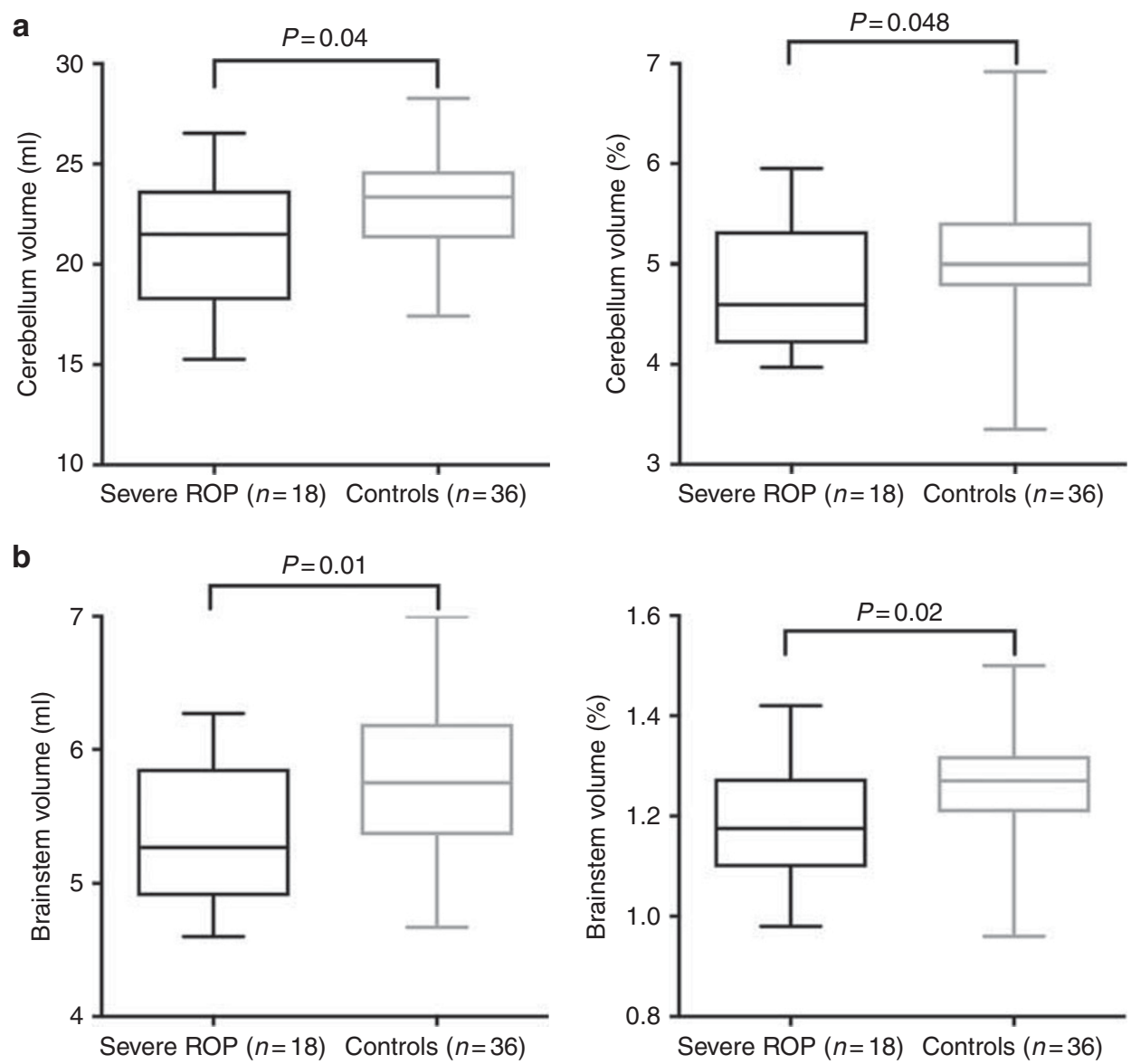

Figure 3. Cerebellum and brainstem volumes. Results of cerebellum and brainstem volumes. Boxplots of the cerebellum (a) and brainstem (b). Left panels show absolute brain volumes and right panels correspond to relative brain volumes. Severe ROP patients showed significantly smaller absolute cerebellar volumes ( $P=0.04$, median: 21.5 (interquartile range: $18.2-23.7 \mathrm{ml}$ ) vs. $23.4(21.3-24.6 \mathrm{ml}), 95 \% \mathrm{Cl}$ : -3.40 to -0.11$)$ and relative cerebellar volumes $(P=0.048,4.6(4.2-5.3 \%)$ vs. $5.0(4.8-5.4 \%), 95 \% \mathrm{Cl}:-0.68$ to -0.007 ; a) as well as significantly smaller absolute brainstem volumes $(P=0.01,5.3(4.9-5.9 \mathrm{ml})$ vs. $5.7(5.4-6.2 \mathrm{ml}), 95 \% \mathrm{Cl}:-0.70$ to -0.10$)$ and relative brainstem volumes $(P=0.02,1.18(1.1-1.3 \%)$ vs. $1.27(1.2-1.3 \%)$, $95 \% \mathrm{Cl}:-0.13$ to -0.007 ; b). $\mathrm{Cl}$, confidence interval; ROP, retinopathy of prematurity.

Table 2. Neurodevelopmental outcome in infancy

\begin{tabular}{|c|c|c|c|}
\hline & $\begin{array}{l}\text { Infants with } \\
\text { severe ROP }\end{array}$ & Control infants ${ }^{\mathrm{b}}$ & $P$ value \\
\hline $\begin{array}{l}\text { Corrected age at GMDS } \\
\text { (months) }\end{array}$ & $\begin{array}{c}15.5 \begin{array}{l}(15.3-16.6) \\
n=15\end{array}\end{array}$ & $\begin{array}{c}15.6(15.2-16.4) \\
n=30\end{array}$ & 0.30 \\
\hline Development quotient & $97(84-104)$ & $101(95-109)$ & 0.01 \\
\hline $\begin{array}{l}\text { Corrected age at BSITD-III } \\
\text { (months) }\end{array}$ & $\begin{array}{c}26(24-30) \\
n=15\end{array}$ & $\begin{array}{c}24(24-27) \\
n=30\end{array}$ & 0.11 \\
\hline Cognitive composite score & $\begin{array}{c}101(91-116) \\
n=15\end{array}$ & $\begin{array}{c}99(88-110) \\
n=30\end{array}$ & 0.15 \\
\hline Fine motor scaled score & $\begin{array}{c}11(10-13) \\
n=12\end{array}$ & $\begin{array}{c}13(10-13) \\
n=24\end{array}$ & 0.02 \\
\hline Gross motor scaled score & $\begin{array}{c}8(8-9) \\
n=11\end{array}$ & $\begin{array}{c}10(8-12) \\
n=22\end{array}$ & 0.15 \\
\hline
\end{tabular}

BSITD-III, Bayley Scales of Infant and Toddler Development assessed at 24 months' corrected age; GMDS, Griffiths Mental Development Scales assessed at 15 months corrected age; ROP, retinopathy of prematurity.

asevere ROP was defined as ROP treated with laser therapy and included ROP grade 3 with plus disease or type 1 ROP.

${ }^{b}$ Control infants without ROP $(n=27)$ or with mild ROP (grade $1 ; n=9$ ). Numbers are displayed as the median (interquartile range).
BS (21). In the cerebellum, IGF-1 promotes development and survival of cerebellar neurons, such as Purkinje cells (22). A number of rodent studies have demonstrated that IGF-1 receptor expression is higher in the cerebellum and BS $(23,24)$. Smaller cerebellum and BS volumes may, therefore, be related to high IGF-1 receptor expression in these structures in preterm infants as well. However, IGF-1 levels were not measured in this study population. Repeating this study by including sequential measurements of IGF-1 serum concentrations to investigate the role of IGF-1 in impaired brain development of ROP patients would be highly relevant.

Alternatively, ROP and brain development may both be susceptible to environmental disturbances because they both proceed at a rapid pace during the preterm period. In addition to low IGF-1 levels, other important factors that could have an impact on brain development include inflammation, infection, hypoxia and hyperoxia, and postnatal malnutrition $(6,25-27)$. 


\section{ROP, brain volumes, and neurodevelopment $\mid$ Articles}

We were able to show that preterm-born children with severe ROP displayed a significantly lower development quotient and lower fine motor scores at 15 and 24 months' CA, which is in agreement with other publications $(28,29)$. Smaller brain volumes and impaired cortical development have been associated with neurodevelopmental deficits that persist through childhood and adolescence (30). Specifically, smaller cerebellar volumes have been associated with poorer cognitive outcome, impaired executive functioning, lower motor performance, and increased risk of autism spectrum disorder (30-33). The cerebellum has increasingly been recognized to be of major importance for cognitive functioning and exerts this role through cerebellar pathways $(25,32,34)$. The cerebellum acts as a control center for higher-order cortical regions (32) and, therefore, early disruptions in cerebellar growth could have an impact on the developing cerebral cortex and in this way affect movement, cognition, and affective regulation (32).

Given the previously described associations between reduced brain volumes and neurodevelopmental outcome, it is particularly relevant to identify infants at risk of long-term neurodevelopmental impairments at an early age (35). Our study underscores the importance of early identification of infants with ROP and the need to examine the brain, including the cerebellum, in these infants. Recently, Davis et al. discussed an overview of neuroprotective therapies that have been proposed in recent years (36). Many of these children develop disturbances of neuromotor development; special education or preventive psychological programs could benefit these children $(35,37)$.

We hypothesized that cortical development would be negatively related to ROP because the cortex develops rapidly in the last trimester of pregnancy (17) and may be susceptible to expected reduced IGF-1 concentrations (9) and other negative environmental and epigenetic influences, potentially associated with severe ROP. However, no association was found in this group with ROP patients. In addition, severe ROP was not related to UWM and CoGM volume in this case-control study. Hence, impaired cortical and white matter development in preterm infants may be related to overall illness severity instead of ROP specifically.

In our study, neonatal morbidity was comparable between infants with ROP and control infants. Nevertheless, erythrocyte transfusions, multiple birth, and BPD were more common in ROP patients. Erythrocyte transfusions were not included in the multivariate model because BPD and erythrocyte transfusions were strongly inter-related. Some studies noted that BPD and multiple birth are related to smaller brain volumes and neurodevelopment $(6,38)$. In the multivariable model of this study, BPD and multiple births had no significant effect on brain volumes and neurodevelopment, suggesting that reduced brain volumes and lower neurodevelopmental scores in ROP patients may be independently related to the pathophysiology and/or possibly the treatment of severe ROP.

Severe ROP could have a negative effect on neurodevelopment because of visual impairment. In this cohort, visual outcome data were available for most of the ROP infants (89\%). Hence, visual impairment could not explain our observations.

There are some limitations that need to be addressed. First, the sample size was small because we only included infants with severe ROP. Fortunately, the incidence of severe ROP is relatively low in our population of extremely preterm infants (39). Because of this small number of infants, our study was not powered to detect all potential changes in neonatal brain growth. Therefore, we underscore that the present study should be repeated in a larger sample. Particularly, regions that are closely connected to the cerebellum may be of interest in such additional studies. A second methodological consideration is that at the time of the analyses not all infants had reached 15 months' or 2 years' CA, and, therefore, we were not able to assess neurodevelopment at this time point in all infants. Nevertheless, medium-term infancy data were available for the majority of infants (83\%) and, therefore, a good estimate of early-life cognitive and motor functioning could be obtained. Long-term neurodevelopmental outcome data at school age, including executive functions, are yet unknown, but are of particular interest with respect to the hypotheses.

In conclusion, we observed smaller cerebellar volumes, cerebellar diameter, and BS volumes in preterm infants with severe ROP compared with matched preterm control infants. Preterm infants with ROP also showed lower overall developmental scores in infancy and poorer fine motor functioning at 2 years' CA. Findings of our study do suggest that preterm-born children with severe ROP are at risk of impaired brain growth of the cerebellum and BS and may be at risk of neurodevelopmental impairment persistent throughout childhood. The underlying mechanism of the association between ROP and early volumetric brain development remains elusive and may be related to alterations in IGF-1 production, which requires further study. In addition, future research is needed to assess the relation of our findings with long-term cognitive and motor functioning.

\section{ACKNOWLEDGMENTS}

We thank Karina Kersbergen, Ingrid van Haastert, and Tabitha Koops for their assistance in the data collection and Professor Margot van Eck van der Sluijs - van de Bor for supervising the first part of this study, which was part of a thesis.

\section{STATEMENT OF FINANCIAL SUPPORT:}

The work of Kristin Keunen is supported by a grant from the Wilhelmina Children's Hospital Research Fund (Vrienden van het WKZ) to Martijn P. van den Heuvel. The work of Floris Groenendaal is supported by a grant from the Netherlands Organization for Health Research and Development (ZonMW; grant number 945-27-022).

Disclosure: The authors declare no conflict of interest.

\section{REFERENCES}

1. Hartnett ME. Pathophysiology and mechanisms of severe retinopathy of prematurity. Ophthalmology 2015;122:200-10. 
2. Hoogerwerf A, Schalij-Delfos NE, Van Schooneveld MJ, Termote JUM. Incidence of retinopathy of prematurity over the last decade in the Central Netherlands. Neonatology 2010;98:137-42.

3. Steinkuller PG, Du L, Gilbert C, Foster A, Collins ML, Coats DK. Childhood blindness. J AAPOS 1999;3:26-32.

4. Hellström A, Smith LEH, Dammann O. Retinopathy of prematurity. Lancet 2013;382:1445-57.

5. Hellström A, Ley D, Hansen-Pupp I, et al. IGF-I in the clinics: use in retinopathy of prematurity. Growth Horm IGF Res 2016;30-31:75-80.

6. Kersbergen KJ, Makropoulos A, Aljabar P, et al. Longitudinal regional brain development and clinical risk factors in extremely preterm infants. J Pediatr 2016;178:93-100 e6.

7. Fernandez AM, Torres-Alemán I. The many faces of insulin-like peptide signalling in the brain. Nat Rev Neurosci 2012;13:225-39.

8. Hellström A, Ley D, Hansen-Pupp I, et al. Insulin-like growth factor 1 has multisystem effects on fetal and preterm infant development. Acta Paediatr 2016;105:576-86.

9. Hansen-Pupp I, Hövel H, Hellström A, et al. Postnatal decrease in circulating insulin-like growth factor-I and low brain volumes in very preterm infants. J Clin Endocrinol Metab 2011;96:1129-35.

10. Hök-Wikstrand M, Hård AL, Niklasson A, Hellström A. Early postnatal growth variables are related to morphologic and functional ophthalmologic outcome in children born preterm. Acta Paediatr 2010;99:658-4.

11. Löfqvist C, Engström E, Sigurdsson J, et al. Postnatal head growth deficit among premature infants parallels retinopathy of prematurity and insulin-like growth factor-1 deficit. Pediatrics 2006;117:1930-8.

12. Papile L, Burstein J, Burstein R, Koffler H. Incidence and evolution of subependymal and intraventricular hemorrhage: a study of infants with birth weights less than 1,500 gm. J Pediatr 1978;92:529-34.

13. Woodward LJ, Anderson PJ, Austin NC, Howard K, Inder TE. Neonatal MRI to predict neurodevelopmental outcomes in preterm infants. N Engl J Med 2006;355:685-94.

14. Kidokoro H, Neil JJ, Inder TE. New MR imaging assessment tool to define brain abnormalities in very preterm infants at term. Am J Neuroradiol 2013; 34:2208-14.

15. Tich SNT, Anderson PJ, Shimony JS, Hunt RW, Doyle LW, Inder TE. A novel quantitative simple brain metric using MR imaging for preterm infants. Am J Neuroradiol 2009;30:125-31.

16. Moeskops P, Viergever MA, Mendrik AM, de Vries LS, Benders MJ, Isgum I. Automatic segmentation of MR brain images with a convolutional neural network. IEEE Trans Med Imaging 2016;35:1252-61.

17. Moeskops P, Benders MJ, Kersbergen KJ, et al. Development of cortical morphology evaluated with longitudinal MR brain images of preterm infants. PLoS ONE 2015;10:e0131552.

18. van Baar M, Steenis AL, Verhoeven LJP. Bayley Scales of Infant and Toddler Development Third. Amsterdam: Pearson Assessment and Information, 2014.

19. Patra K, Greene MM, Patel AL, Meier P. Maternal education level predicts cognitive, language, and motor outcome in preterm infants in the second year of life. Am J Perinatol 2016;116:1477-90.

20. Harrel S, Brandon D. Retinopathy of prematurity: the disease process, classifications, screening, treatment, and outcomes. Neonatal Netw 2007;26: 371-8.

21. Moore JK, Linthicum FH. The human auditory system: a timeline of development. Int J Audiol 2007;46:460-78.

22. Ye P, Xing Y, Dai Z, Ercole AJD. In vivo actions of insulin-like growth factor-I (IGF-1 ) on cerebellum development in transgenic mice: evidence that IGF increases proliferation of granule cell progenitors. Brain Res Dev Brain Res 1996;95:44-54.

23. Popken GJ, Hodge RD, Ye P, et al. In vivo effects of insulin-like growth factor-I ( IGF-I ) on prenatal and early postnatal development of the central nervous system. Neuroscience 2004;19:2056-68.

24. Dentremont KD, Ye P, Ercole AJD, O’Kusky JR. Increased insulin-like growth factor-I (IGF-I) expression during early postnatal development differentially increases neuron number and growth in medullary nuclei of the mouse. Dev Brain Res 1999;114:135-41.

25. Limperopoulos C, Soul JS, Gauvreau K, et al. Late gestation cerebellar growth is rapid and impeded by premature birth. Pediatrics 2005;115: 688-95.

26. Lofqvist C. Postnatal head growth deficit among premature infants parallels retinopathy of prematurity and insulin-like growth factor-1 deficit. Pediatrics 2006;117:1930-8.

27. Keunen K, Išgum I, van Kooij BJM, et al. Brain volumes at termequivalent age in preterm infants: imaging biomarkers for neurodevelopmental outcome through early school age. J Pediatr 2016;172: 88-95.

28. Beligere N, Perumalswamy V, Tandon M, et al. Seminars in fetal \& neonatal medicine retinopathy of prematurity and neurodevelopmental disabilities in premature infants. Semin Fetal Neonatal Med 2015;20: 346-53.

29. Glass TJA, Chau V, Gardiner J, et al. Severe retinopathy of prematurity predicts delayed white matter maturation and poorer neurodevelopment. Arch Dis Child Fetal Neonatal Ed 2017;102:F532-7.

30. Lind A, Haataja L, Rautava L, et al. Relations between brain volumes, neuropsychological assessment and parental questionnaire in prematurely born children. Eur Child Adolesc Psychiatry 2010;19:407-17.

31. Anderson P, Doyle LW. Neurobehavioral outcomes of school-age children born extremely low birth weight or very preterm in the 1990s. J Am Med Assoc 2003;289:3264-72.

32. Stoodley CJ, Limperopoulos C. Structure-function relationships in the developing cerebellum: evidence from early-life cerebellar injury and neurodevelopmental disorders. Semin Fetal Neonatal Med 2016;21:356-64.

33. Limperopoulos C, Bassan H, Sullivan NR, et al. Positive screening for autism in ex-preterm infants: prevalence and risk factors. Pediatrics 2008; 121:758-65.

34. Van Kooij BJM, Benders MJNL, Anbeek P, Van Haastert IC, De Vries LS, Groenendaal F. Cerebellar volume and proton magnetic resonance spectroscopy at term, and neurodevelopment at 2 years of age in preterm infants. Dev Med Child Neurol 2012;54:260-6.

35. Cioni G, Inguaggiato E, Sgandurra G. Early intervention in neurodevelopmental disorders: underlying neural mechanisms. Dev Med Child Neurol 2016;58:61-.

36. Davis AS, Berger VK, Chock VY. Perinatal neuroprotection for extremely preterm infants. Am J Perinatol 2016;33:290-6.

37. McGrath JM, Cone S, Samra Ha. Neuroprotection in the preterm infant: further understanding of the short- and long-term implications for brain development. Newborn Infant Nurs Rev 2011;11:109-2.

38. Neubauer V, Junker D, Griesmaier E, Schocke M, Kiechl-Kohlendorfer U. Bronchopulmonary dysplasia is associated with delayed structural brain maturation in preterm infants. Neonatology 2015;107:179-84.

39. van Sorge AJ, Termote JUM, Simonsz HJ, et al. Outcome and quality of screening in a nationwide survey on retinopathy of prematurity in The Netherlands. Br J Ophthalmol 2014;98:1056-60. 